\title{
Q1 Social Studies of Nanopharmacy Nanopharmaceutical Research
}

Michael Schillmeier

University of Exeter, Department of Sociology \& Philosophy, Byrne House, St German's Road, Exeter EX4 4PJ UK

21.1

Engaging with Ethical, Legal, and Social Implications of Nanoresearch

Since the Human Genome Project in the 1990s, ELSI accounts (i.e., the discussions of ethical, legal, and social implications of science and technology) have gained substantial importance and have drawn attention to the "coproduction" [1] of science, technology, and society [2]. ELSI accounts echo a long tradition of engaging with social, historical, political, and philosophical issues regarding the development of science and technology. Moreover, since the late 1970s, "science and technology studies" (STS) have been involved in understanding the concrete problems, questions, and issues of research practices [3]. Clearly, STS and ELSI accounts play an important role in understanding the different and changing ways of how science and technology both shape and are shaped by societal organization, cultural production, and related interests and values [4].

With the emergence of nanoscience and nanotechnology, STS and ELSI accounts have discussed in detail the ethical and social issues of "nano" as well as the importance of public understanding and engagement with science and technology [5]. It is argued that "ethical considerations are the foundations of healthy progress, and must be openly participatory in their undertaking." [6] However, due to the specificities of nano and nanopharmacy, current ethical studies do not share a common perception of what the exact ethical issues are [7]. Moreover, it remains undecided if nanopharmacy challenges the canon of bioethical issues as they can be found in other emerging fields within the life sciences [8]. General ethical issues like patient's rights, autonomy and responsibility, responsible innovation, risk/benefit analysis in clinical trials, issues of missing definitions, standardization and characterization, global challenges of regulation [9], human enhancement, misuse of medical technologies and bioterrorism, environmental safety, and so on dominate current ethical debates and

Pharmaceutical Nanotechnology: Innovation and Production, First Edition. Edited by Jean Cornier, Arno Kwade, Andrew Owen, and Marcel Van de Voorde.

(c) 2017 Wiley-VCH Verlag GmbH \& Co. KGaA. Published 2017 by Wiley-VCH Verlag GmbH \& Co. KGaA. 
are hardly novel concerns. Still, it is important not to act as if ethical issues of nanopharmacy can be deferred to more general debates about bioethics. Ethical issues are part of the specificity of every research agenda, the more so if it is governed by a myriad of uncertainties and possible risks as is the case with nanotechnological research [10].

A crucial set of ethical issues, next to the ones briefly mentioned above, concerns the questions of higher costs, affordability, equal accessibility, and distribution of clinically available nanomedicines. Hall et al. [11] have argued that "the higher costs of the clinically available nanodrugs may be attributed more to the fact that they are "newer" and more effective than their pharmaceutical principles alone, with the added manufacturing costs playing a minor role." Although an ethical problem in itself, it poses a more general ethical question concerning the affordability, accessibility, and distribution of nanopharmaceuticals. I suggest that a general ethos should foster the idea of "good" nanopharmacy. The ethos I propose could read as follows: What appears as good nanopharmacy for the poor is equally good for those who are better off, or consider themselves more privileged. Moreover, nanopharmacy also needs to engage with the limits of the ethics of Big Pharma as well as the problem of "institutional corruption and the pharmaceutical industry" [12].

\section{2}

\section{Nanopharmacy and the "Culture of Promise"}

The French philosopher of science and mathematician Michel Serres has argued that the object of science is not a given, but it is science that creates its objects and, if necessary, desists from them. The objects of science generate the "horizon of knowledge" and related research processes and practices that define what kind of objects become part of science and which are excluded. Moreover, as we know from the history of pharmaceutical research, even a well-functioning medicine is never a finished "best" product, but is always open for improvement. Thus, in many ways the scientific "object is project" as Serres has put it bluntly [13].

Undoubtedly, the objects of nanopharmacy (as well as the question of ethics) are very much projects. In the case of chemotherapeutics, for instance, such projects span a horizon of innovative pharmacological thoughts from the late nineteenth century up to now. This can be seen in Paul Ehrlich's idea of targeted drug delivery via a "magic bullet," [14] which was advocated in 1906 and still plays a vital role in imagining current project-objects in nanopharmacy [15].

With the "project-objects" in pharmacy comes a "culture of promise" to provide medical and pharmaceutical improvements and benefits for the treatment of diseases [16]. The "culture of promise" gained temporary climax by highlighting the revolutionary prospect of delivering drugs and nanomedicines measurable in a nanometer size range. It was meant to lead to improved pharmacokinetics and 
pharmacodynamics, which in turn ought to enable more specific, efficient, less toxic, and lower cost medicines for the majority of diseases and their multiple side effects. Although improvements in pharmacological research and related visions contribute to effective healthcare practices, the excessive culture of promise in nanotechnology research have set unrealistic targets and even have been counterproductive for nanopharmaceutical research itself if the promised results and goals could not be met.

This asymmetry between promise and actuality creates a problem that risks becoming a source of distrust in nanopharmaceutical research. The more so if it promotes an unconsidered and iterative slogan of "fast science" and its science policies that make absent concrete problems and issues of specific research investigations on a nanoscale [16]. Hence, the "culture of promise" is not just an innocent attribution of the "goodwill" of science, but also deeply affects how research is carried out as a matter of fast science. The latter comprises the shortening of research projects, the pressure of the "publish or perish" ideology, the dominant interest of publishing merely positive results and obscuring negative ones or "dead ends," the increasing power of economic reasoning that governs scientific research practices, and so on.

All these requirements of fast science may be at odds with the demands of what I like to call "good translation" in nanopharmacological research. Good translation includes, for instance, precise characterization of a new drug formulation as well as the reproducibility of an experimental design to meet the standards of a stable and consistent medicine, but also the full recognition that nanopharmacy is not an independent research activity but assembles a wide range of scientific and nonscientific actors. All these actors need to gain an equal stake in nanopharmacy. In addition, all procedures and interactions with heterogeneous actors and their interest and expertise require a slowing down rather than a speeding up of scientific practices. Unfortunately, however, the agenda of fast science and the related "culture of promise" have nothing creative to contribute. On the contrary, this prevailing policy reinforces the fierce rules of accelerating and intensifying global competition rather than aiming at good translation. To be sure, competition is good as long as it allows us to respond to and address, in a responsible way, the requirements of novel fields of research such as nanopharmaceutical agendas, in which the application of good pharmaceutical solutions to a medical issue is an ongoing project rather an accomplished goal.

21.3

From "Science Meets Society" to Translation as a Social Process

Responsible research has to focus on improving the translational processes of nanopharmaceutical medicines for all actors involved - from bench to bedside. This also means that the notion of "translation," which focuses on applied 
research in healthcare and gained widespread recognition in fast science accounts, needs reconsideration. First, and most importantly, the notion of translation should not be conflated with a smooth process of linear progression or "best way" of research development. Rather, we find at every step of nanopharmaceutical research different processes of translation that can be better understood as complications that arise from their application. Complications start with the prospect of immature and often unrealistic ideas that turn into a concrete research question, which may develop into preclinical in vitro experimentation that, if the researcher is lucky, may translate into in vivo experimentation. This may further translate into a cascade of clinical trials and, if successful, into an approved nanomedicine, which then translates into a prescription of a drug that translates into different effects while interacting with different and changing physiology as well as (possibly) with other prescribed drugs. This long and interlaced sentence portrays awkwardly but adequately the high probability that ideas may not translate into applicable end products. However, it also tells us that there is no way of cutting this process short if we wish to improve given medicines.

Moreover, the nanopharmaceutical process of translation relates cognitive objects (ideas) with material experimentation, molecular processes with cells and organisms, animal models with human bodies, the laboratory with other laboratories, assets with computers, pharmacology and toxicology [17], the laboratory with the clinic, laboratory experimentation with highly specific patient needs, the characterization of nano-objects with regulatory challenges and requirements, nanomaterials with ethical challenges and philosophical reflection, pharmaceutical efficacy with economic reasoning and political interests, and so forth. It assembles what I like to call the social complexity of nanopharmacy, which relates and composes heterogeneous processes and actors, requirements, and obligations [18]. Moreover, each process of translation may feed back on earlier stages of translation and alter their understanding and composition. Untranslatability or failures of translation may provoke novel forms of experimental practices that suggest new forms of translation that may entail unexpected results or undesirable side effects.

Hence, each process of translation links as well as creates different processes and materialities, different requirements, different obligations, and different trials that decide upon the quality of the research project-object and its future. Thus, translation refers to a set of practices that constantly alters the processes and objects involved. Each phase of translation presents a complication and thus adds complexity to the overall process of nanopharmaceutical research and usage. It assembles a process of multiple translations, which although not comparable, aim to compose compatible relations across and with different scales of action (nano/micro/macro).

It is of the utmost importance that social studies of nanopharmacy engage with and analyze the myriad of translations as they become apparent in current research activities. Accordingly, social studies of nanopharmacy are not restricted to the rather abstract and misleading idea of "science meets society" as 
put forward by the rhetoric of fast science, as if "science" and "society" refer to two different spaces of reality. It doesn't help "science" much when merely informing the nonscientific audience about work in order to gain trust and recognition. As Weitze and Heckl [19] have argued, the corresponding idea of "public understanding of science," which suggests that society lacks understanding and thus "proper information and explanation could bring together science and society," has been "proven to be empirically wrong." It appears similarly abstract and misconceived to argue that it is the society that needs to critically assess the effects of science from the outside.

The idea of "public engagement with science" advocates a different route, which is not about science, but a way to collaborate, practice, and experiment with science, although the experimental practices differ from the laboratory experimentation. As I have argued elsewhere, the concept of "publics," as envisaged by authors like John Dewey, Walter Lippman, and others, offers an innovative way of engaging with scientific practice and emerging technologies, linking lab experimentation with all the relevant actors (e.g., patients, clinicians, researchers, regulators, concerned citizens, NGOs, policy makers, industry, and so forth) who are concerned with a specific nanomedical or nanopharmaceutical issue:

Nanomedical publics bring together people (and their practices and objects) who share a concern for an issue for which no immediate answers and fixed solutions are given (or can be expected). Publics, then, engage with the ambiguities and uncertainties of scientific research practices, their relevant objects of concern and their possible effects inside and outside the scientific laboratory [20].

Generally speaking, social studies of nanopharmacy are obliged to care about all the different processes of translation as they may occur in the development and usage of nanomedicines. In that sense, social studies of nanopharmacy involve the analysis and collaboration with all the diverse actors - ideas and materials, human and nonhuman, scientific and social scientific, scientific and nonscientific, bodies and technologies, the clinic and patients - as necessary mediators, who/which affect and are affected by nanopharmaceutical research.

21.4

Metaphors and Nanopharmacy

Social studies of nanopharmacy also suggest that the way we imagine and talk about research issues and objects has a significant impact on how nanomedicines are produced as well as how health and illness is understood. Current studies of nanopharmacy underline that the prevailing idea of the "magic bullet" and the related "culture of promise" of nanopharmacy and nanomedicine mirror "the engineering values of control, precision, efficiency, and surveillance" which are 
"dominantly framed in terms of military metaphors" [21-23]. STS scholar Sacha Loeve states that

in the current context of crisis of pharmaceutical innovation, military metaphors do provide a persuasive means to sustain a climate of 'general mobilisation' similar to wartime. In using them, researchers, start-ups, clinicians and patients may feel that despite their diverse stakes and interests, they take part in a crusade in which they are all united - both the crusaders and the crusaded (i.e. patients) - against a common enemy [24].

For Loeve, military or ballistic metaphors are poorly chosen to account for the different processes of how nanoparticles interact with changing environments. They underestimate the body being an active, dynamic, and heterogeneous system. The military metaphor not only transports a rather under-complex understanding of disease and the body, it also configures the patient as a "fighter" and thus plays a powerful, but also ambivalent description both of experiencing illnesses (like cancer) and its modes of medical engagement [25]. However, Loeve also notes that current nanopharmaceutical and nanomedical research agendas are informed by a change from military metaphors toward "ecological" metaphors as well as a wide range of nonmilitary metaphors used by patient experiences of their illness ("journey," "dance," "drama"). Hence, the multiplication of metaphors refers (1) to more complex, diverse, and individually experienced understandings of disease and the body, (2) to the social and cultural dimensions of illness, (3) to the changing medical ways of dealing with and caring about disease, and (4) to an understanding of nanopharmaceutical objects as milieudependent medicines [26,27].

In such a reading, metaphors in nanopharmacy are historically changing tools of understanding, shaping, and engaging with health and illness in research and clinical practices. Thus, the employment of different metaphors mirrors and evokes different developments and cultures of nanomedicines [28,29]. They may contribute to a more complex and thus more realistic understanding of illness, related processes of interacting human bodies, and the relevance of changing and diverse (inner and outer) environments. Clearly, the ideas of "being at war with" or the "magic bullet" offer rather under-complex and limited imaginaries to do so. Vincent and Loeve [30] rightly stress that "addressing drugs to a specific site and releasing the right dose in the right place at the right moment in the complex environment of a living body requires more than a magic bullet."

21.5

Nanopharmacy and "Personalized Medicine"

The nanopharmaceutical promise entails the prospect of moving from the development of highly standardized "block buster" and "one size fits all" pharmaceutics toward targeting the precise nanomedical formulas and doses that reflect the 
individual situation of illness(es), its differences in disease emergence and its idiosyncratic responses to treatments.

This promised relationship between nanopharmaceutical development and personalized medicine is thought to promote and further unfold a new "episteme" [31] in medicine, that is, a way of locating and understanding diseases and patients as well a the fundamental reorganization of healthcare in general $[32,33]$. However, as the STS scholar and philosopher Xavier Guchet and others have shown recently, the relationship between nanomedicine, nanopharmacy, and personalized medicine is not straightforward. Rather, it assembles polysemic understandings and conflicting expectations of/from personalized medicine and articulates controversies over the concrete meaning of "person" and "personalization" within biomedical research agendas [34]. Moreover, personalized nanopharmacy needs to pay attention to the increasing importance given to research findings in epigenetics, which highlight the significance of different and shifting human/environment relationships in understanding individual disease formation and their effects [35]. Up to now, it remains unresolved whether the progress in personalized nanopharmacy will compliment and improve novel developments of "patient-centered" forms of care or if it merely extends the current trend of the technomolecular gaze in medicine and, thus strengthens the biomedicalization and individualization of disease at the expense of social and patient centered models of healthcare.

The future of personalized nanopharmacy is imagined to "enhance[s] the autonomy of the patient and user by giving them more control, but at the same time shifts responsibility and liability to the service providers and product developers as the medical professionals slowly move out of the picture" [36,37]. Such a technologically mediated individualism unfolds the neoliberal figure of the competent and self-responsible individual. In effect, medical power becomes partly disentangled from the medical profession and is instead distributed among technological and market-driven networks, as well as clever and autonomous individuals [37]. Clearly, such a technocratic perspective through which the patient becomes a rational "user" of technologies and the "client" of service providers not only creates the neoliberal myth of the autonomous patient, but also presupposes the autonomous, rational, capable, and well-connected individual.

By installing the "healthy," able, and autonomous person, the image of technologically mediated pharmacy and medicine makes absent the everyday life of experiencing illness and the associated requirements and obligations. The prevailing argument that nanotechnological advances in medicine and pharmacy shift the responsibility from the medical system to the individual who is thereby thought to gain autonomy, is a rather problematic assumption of facets of a neoliberal discourse that requires the "autonomous subject" for its reasoning and economy driven strategies. It also assumes a rather simplistic, one-sided, and normative understanding of technology as a fixed tool of improvement and enhancement. By making this point clear I do not wish to downplay the importance of patients gaining their own voice or the possibilities of technological advance in pharmacy and healthcare settings. Quite the contrary, the future of 
personalized nanopharmacy cannot do without a shift toward an in-depth view of the situated and specific requirements of patients' needs as well as the perspectives and experiences of the different patients. We need to empower patients and patient groups to engage with medical and pharmacological research in the prevention, diagnosis, and treatment of diseases. Cleary, the engagement of patients with nanopharmacological research and application is not well established and needs novel spaces of interaction between patients, interested citizens, research agendas, medical practice, the pharmacy, and the clinic. It needs to be observed with vigilant eyes if and how strategies of propagating biomolecular understandings of health and illness will create empowering experiences of the individual or if it just names the shift from medical to more technocratic forms of power.

Although the patients play an important role in the current nanomedical rhetoric, they rarely play an active part in the nanopharmaceutical research processes. Rather, they remain merely vulnerable individuals in the course of clinical trials and clinical practices. They may also become victims of disillusion suffering from a "culture of promise" that promises unrealistic results that cannot be achieved in a short period of time. The constructive role of patients and patient advocacy groups is vital, precisely when (object and technologically mediated) risks and uncertainties dominate the diagnosis and the treatment of diseases with nanopharmaceuticals that not only treat diseases, but also unfold a wide range of uncertainties and risks [38]. Emerging risks and uncertainties that cannot be adequately dealt with by the prevailing rationalist model of risk/benefit analysis of science troubles the relationship between patient, nanopharmaceutical treatment, and the classical idea of informed consent. As Timmermanns et al. [39] have pointed out: "As long as nanopharmaceuticals are associated with risks, but also with significant uncertainty and ignorance ('unknown unknowns'), it may prove as an impossible task to inform affected persons adequately."

In such a situation where middle- and long-term effects and possible acute individual side-effects may be unknowingly produced by pharmaceuticals, for which nanopharmacy can not offer a reliable risk/benefit analysis and established treatments, the individual (or a subgroup) becomes the bearer of "second order risks" that escape the scientific reasoning of calculable probabilities [40]. Second order risks are those uncertainties and risks that are produced by the progress of our current institutions, for which at the same time no institutionalized remedies are available if they produce adverse effects.

21.6

Concluding Remarks

Although there is a long-standing and growing body of fine empirical and conceptual studies on science and technologies including the social studies of nanoscience, nanotechnology, and nanomedicine, it should be critically noted that scientists have little awareness of these literatures. Cooperations between the 
different fields of nanoresearch and STS or ELSI projects are primarily motivated by current requests for grant applications and directives of science policymakers and rarely born directly from research issues and problems. It should also be noted that the idea of converging technologies and the demand for transdisciplinary work, necessary for an adequate understanding of nanoscaled processes in medicine and pharmacy, does not include social sciences and STS. However, it seems to me that the time is more than ripe for transdisciplinary research agendas to include from the very beginning the cooperation with social sciences, and invest in diverse forms of public engagement [41]. The engagement of and with a plurality of actors - scientific and nonscientific - should not be seen as an obstacle but, rather, should be internalized as the ultimate basis for a collectively responsible process of scientific experimentation. Nanopharmacy can play a pioneering role in rethinking scientific practices understood as a social accomplishment that engages in the inclusion of all relevant and interested actors involved in emerging healthcare technologies and novel research practices.

\section{References}

1 Jasanoff, Sh. (ed.) (2004) States of Knowledge: The Co-Production of Science and the Social Order, Routledge, London.

2 Schillmeier, M. (2015) What ELSI/A makes big and small in nanotechnology research, in Absence in Science, Security and Policy: From Research Agendas to Global Strategies (eds B. Rappert and B. Palmer), Palgrave Macmillan, Houndmills, UK, pp. 55-77.

3 (a) Knorr-Cetina, K. (1995) Laboratory studies: the cultural approach to the study of science, in Handbook of Science and Technology Studies (eds Sh. Jasanoff et al.), Sage, London, pp. 140-166. (b) Latour, B. (1987) Science in Action: How to Follow Scientists and Engineers through Society, Harvard University Press, Cambridge, MA. (c) Latour, B. (1988) The Pasteurization of France, Harvard University Press, Cambridge, MA. (d) Latour, B. and Woolgar, St. (1986) Laboratory Life: The Construction of Scientific Facts, Princeton University Press, Princeton (originally published in 1979).

4 (a) The literature is vast, but for an introduction, see, for example, Gibbons, M., Limoges, C., Nowotny, H. Schwartzmann, S., Scott, P., and Trow, M.
(1994) The New Production of Knowledge: The Dynamics of Science and Research in Contemporary Societies, Sage, Los Angeles. (b) Nowotny, H., Scott, P., and Gibbons, M. (2001) Rethinking Science, Knowledge and the Public in the Age of Uncertainty, Polity, London. (c) Keller, E.F. (2002) The Century of the Gene, Harvard University Press, Harvard. (d) Rheinberger, H.J. (2010) An Epistemology of the Concrete: Twentieth-Century Histories of Life, Duke University Press, Durham.

5 (a) For a brief but critical summative discussion, see Schillmeier, M. (2015) What ELSI/A makes big and small in nanotechnology research, in Absence in Science, Security and Policy: From Research Agendas to Global Strategies (eds B. Rappert and B. Palmer), Palgrave Macmillan, Houndmills, UK, pp. 55-77.

(b) For a specific focus on nanomedicine, see Schillmeier, M., Barz, M., and Luxenhofer, R. (2015) Quo vadis nanomedicine? Nanomedicine, 10 (20/21), 3089-3091.

6 Hall, R.M., 2 ., and Ferrari, M. (2012) A portrait of nanomedicine and its bioethical implications. J. Law Med. Ethics, 14 (4), 763-779. 
Q5 7 (a) Timmermans, J., Zhao, Y., van den Hoven, J. (2011) Ethics and nanopharmacy: value sensitive of new drugs. Nanoethics, 5, 269-283. (b) See also Costa, H.S., Sethe, S., Pego, A.P., and Olsson, A.S. (2011) Scientists' perception of ethical issues in nanomedicine. Nanomedicine, 6 (4), 681-691. (c) Kazemi, A., Majidinia, M., and Jamali, A.A. (2014) The question of ethics in nanomedicine. J. Clin. Res. Bioeth., 5 (4), 193. (d) Resnik, D.B. and Tinkle, S.S. (2007) Ethics in nanomedicine. Nanomedicine, 2 (3), 345-350.

8 Kuiken, T. (2011) Nanomedicine and ethics: is there anything new or unique? Nanobiotechnology, 3, 111-118.

9 Bowman, D.M. and Gatof, J. (2015) Reviewing the regulatory barriers for nanomedicine: global questions and challenges. Nanomedicine, 10 (21), 3275-3286.

10 See, for example, the symposium: Wolf, S.M., McCullough, J., Kahn, J.P., Jones, C. (2012) (eds) Nanodiagnostics and nanotherapeutics: building research ethics and oversight. J. Law Med. Ethics, 40 (4)

11 Hall, R.M., Sun, T., and Ferrari, M. (201 Nanomedicine and its bioethical implications. J. Law Med. Ethics, 40 (4), 776.

12 See, for example, the special issue Institutional corruption and the pharmaceutical industry. J. Law Med.

Q4 Ethics, 41 (3)

13 Serres, M. (1) Iermes II: Interferenz, Merve, Berlin, p. 81 (originally published in 1972).

14 Ehrlich, P. (1960) Address delivered at the dedication of the Georg-Speyer-Haus, in The Collected Papers of Paul Ehrlich (ed. F. Himmelweit), Pergamon Press, London, pp. 53-63 (originally published in 1906).

15 Schillmeier, M., Barz, M., and Luxenhofer, R. (eds) (2015) Quo vadis nanomedicine? Nanomedicine, 10 (20/21), 3089-3091.

16 Schillmeier, M. (2015) Caring about social complexity in nanomedicine.

Nanomedicine, 10 (20), 3181-3193.

17 See, for example, PourGashtasbi, G. (2015) Nanotoxicology and challenges of translation. Nanomedicine, 10 (20), 3121-3129.
18 Schillmeier, M. (2015)

19 Weitze, M.-D. and He $2 . M$. (2015) Communicating science in the making challenges and opportunities. Nanomedicine, 10 (20), 3099.

20 Schillmeier, M. (2015) 3189.

21 Loeve, S. (2015) Of drug administration, war and oïkos. Mediating cancer with nanomedicines. Nanomedicine, 10 (21), 3261-3274.

22 Loeve, S., Vincent, B.B., and Gazeau, F. (2013) Nanomedicine metaphors: from war to care. Emergence of an ecological approach. Nano Today, 8 (6), 560-565.

23 See, for example, Franzen, S. and Lommel, S.A. (2009) Targeting cancer with 'smart bombs': equipping plant virus nanoparticles for a 'seek and destroy' mission. Nanomedicine, 4 (5), 575-588.

24 Loeve, S. (2015)

25 Loeve, S. (2015)

26 Loeve, S. (2015)

27 Vincent, B.B. and 2 e, S. (2014) Metaphors in nanomedicine: the case of targeted drug delivery. Nanoethics, 8 (1), $1-17$.

28 Annas, G.J. (1995) Reframing the debate on health care reform by replacing our metaphors. N. Engl. J. Med., 332 (11), 744-747.

29 Camus, J.T.W. (2009) Metaphors of cancer in scientific popularization articles in the British press. Discourse Stud., 11 (4), 465-495.

30 Vincent, B.B. and Loeve, S. (2014) Metaphors in nanomedicine: the case of targeted drug delivery. Nanoethics, 8 (1), $1-17$.

31 Foucault, M. (1973) The Birth of the Clinic. An Archaeology of Medical Perception, Tavistock Publications Limited, London.

32 Weston, A.D. and Hood, L. (2004) Systems biology, proteomics, and the future of health care: towards predictive, preventive, and personalized medicine introduction: paradigm changes in health care. $J$. Proteome Res., 3, 179-196.

33 Guchet, X. (2015) 'What's in a word? The person of personalized (Nano) medicine. Nanomedicine, 10 (20), 3167-3179.

34 (a) Guchet, X. (2015 Dee also Pokorska-Bocci, A., ht., Sagoo, 
G.S., Hall, A., Kroese, M., and Hillary, B. (2014) Personalized medicine: what's in a name? Personal. Med., 11 (2), 197-210. (c) Tutton, R. (2012) Personalizing medicine: futures present and past. Soc. Sci. Med., 74, 1721-1728.

35 Chadwick, R. and O'Connor, A. (2013) Epigenetics and personalized medicine: prospects and ethical issues. Personal. Med., 10 (5), 463-471.

36 Timmermans, J., Zhao, Y., and Hoven, J.v. d. (2011) Ethics and nanopharmacy: value sensitive of new drugs. Nanoethics, 5, 279.

37 Kuiken, T. (2011) Nanomedicine and ethics: is there anything new or unique? Nanobiotechnology, 3, 111-118.
38 See in the case of HIV and other diseases Callon, M., Lascoumes, P., and Barthe, Y. (2001) Acting in an Uncertain World: An Essay on Technical Democracy, The MIT Press, Cambridge/MA./London, England.

39 Timmermans, J., Zhao, Y., and van dan Hoven, J. (2011) Ethics and nanopharmacy: value sensitive of new drugs. Nanoethics, 5, 274.

40 On second order risks' that unfold beyond the modernist framework of calculative probabilities, see Beck, U. (1992) Risk Society: Towards a New Modernity, Sage, London.

41 See, for example, Schillmeier, $M$. (2015)

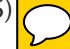




\section{Author Query}

1. Please check the chapter title.

2. References $5 b$ and 15 are indentical. Please check and suggest.

3. Please provide the page range for Reference 10.

4. Please provide the author/authoring organization, year, volume number and page range in Reference 12.

5. Please provide book/journal title and other bibliographic details for References $18,20,24,25,26,34 a$ and 41 . 